\title{
Impact of physical exercise in developing some motor abilities and improving performance of some basic motor skills for mentally disabled.
}

\section{Eslam Mohamed Salim}

Ass. prof , Exercises and Gymnastics Training Department, Faculty of Sport Education for Men, Alexandria University. Egypt.

\section{Wael Kamel Al Hawy}

Lecturer, Exercises and Gymnastics Training Department, Faculty of Sport Education for Men, Alexandria University. Egypt.

\section{Mohamed Abdel Fattah Moghazy}

Ass. Lecturer, Exercises and Gymnastics Training Department, Faculty of Sport Education for Men, Alexandria University. Egypt.

\begin{abstract}
Nations progress and development is measured by what they offer to handicapped from therapeutic, educational, rehabilitative and recreational services, because they, like other community members have a right to life, growth, education and training for a career to be productive in society, Mental disability problem have a great concern; because it is a problem associated with mental efficient to humans, especially that any society depends on its development and progress on mental competency of its members, and hence it is problem of a special nature and versatile, which make it a unique model, not similar with any other disability problem. Identifying Impact of physical exercise in developing some motor abilities and improving performance of some basic motor skills for mentally disabled pupils (8-12 years). Experimental approach was used for its suitability to research nature. This study was conducted on a sample of mentally disabled students in age stage (8 -12 years) with mental age ranges between (4-6) years and IQ range between (50 - 75) degrees, Pilot studies and pre-measurements in the period from 26/01/2013 to 07/02/2013, and main experiment in the period from 09/02/2013 to 04/04/2013, and post-measurements in the period from 06/04/2013 to 08 / 4/2013. Saad Zaghloul and Ikhlas mental education schools, West Educational Department, Alexandria governorate and main study conducted on intentionally selected sample from mentally disables pupils from Saad Zaghloul mental education school, totaled to (20) students, divided into two groups, one experimental, and the other control each of (10) pupils, while the pilot studies conducted on intentionally selected sample from mentally disables pupils from Ikhlas mental education school totaled to (12) pupils. Results of the research are: 1) Experimental group which followed the proposed training program of physical exercise surpassed control group, 2) Developing motor skills for mentally disabled pupils have a positive impact in improving basic motor skills (Walking - running - throwing catching-pushing- balance), 3) Mentally disabled pupils practice building and special purpose exercises with or withut using tools and by following scientific foundations when implemented resulted in improved motor skills for pupils with mental disabilities. At last we recommend the following points: 1) Physical exercise program to be applied to mentally disabled pupils through specialized Physical Education graduates, 2) It is necessary when planning mental schools programs to develop physical abilities programs and implement it for mentally disabled students because of its positive impact in improving performance of basic motor skills.
\end{abstract}


Introduction:

$\mathrm{N}$ ations progress and development is measured by what they offer to handicapped from therapeutic, educational , rehabilitative and recreational services, because they, like other community members have a right to life, growth, education and training for a career to be productive in society. Mental disability problem have a great concern; because it is a problem associated with mental efficient to humans, especially that any society depends on its development and progress on mental competency of its members, and hence it is problem of a special nature and versatile, which make it a unique model, not similar with any other disability problem.

Yossr Abdel Ghani (2001) and Mohamed Fathy (2004) states that mentally disabled children have a lot of delays in acquiring basic motor skills compared with normal children. They also characterized by slow movement, difficulties in coordinating fine movements, disorders in direction organizing (right - left- front - back), lack of motor coordination, inability to balance while walking, as well as irregular breathing.(1:12) (27:5)

According to Christine McIntyre (2004) mentally disabled child could not acquire skill with a high degree like normal children, so it should be emphasized on motor skills learning like correct standing, walking, running jump and hung up, as essential movements of environmental adaption, with an attempt to teach him some special sport motor skills tailored to his case, and which does not require many knowledge or a high coordination between body parts, and at the same time working to increase physical fitness level and improve muscle tone.(20:117)
Gomaa Sobhy (2006) indicates that the child who do not arrive to good performance of basic motor skills will later face a big problem in acquiring sport skills, so it must be concerned with in motor programs kinetic for child during this age stage in order to gain new skills, which in turn are divided into (transitions - non transition movements movements of processing and handling). (28:17)

Khairia ElSokary et al (2005) argue that that the importance of basic motor skills for children with mantal disabilities represented in (work on strengthening the body's vital systems like muscle and respiratory system, balanced growth for body organs, raise general health status, self-reliance in doing their different needs, leading to rise of social level). (12:126-127)

Amal Alhjursy (2002) indicated that mentally disabled individual is delayed in physical fitness then his normal peers, and in lower motor performance of sophisticated motor skills like balance, transitional movements and manual skills when compared normal peers in same age group.(4:220)

Hassan Alnoasrh (2010) indicates that physical education programs for this category do not differ from programs to normal students, but disability represents slower in learning process, in addition to that mentally handicapped students have low performance of body mechanics, so attention must be given to physical and skill activities programs in order to help on the growth of motor, social skills and psychological skills, it is quite possible that mentally handicapped children will be weak in physical and motor fitness compared to their peers normal peers; due to avoid participation in some activities, and is also likely to be weak in motor coordination and balance.(6:159) 
Ministry of Education in teacher's manual of preparation phase (2003) states that everyone has the right to have effective role in his community, and disability should not be cause in deprivation of this right, there is necessity for giving attention to mentally disabled children and invest their capabilities ensure their adaption with environment surrounding them, and prepare them for incorporation with their community members, as well as care for enrichment and development programs suit nature of their disability as a natural requirement and essential guidance in raising these children and helping them to interact with their environment. That is push us to put their own charters, and provide them with more benefits than we provide to their normal peers. (21:34)

Basic motor skills are divided to transitional movements (walking, running, high and long jump, and leap), non-transitional movements (bending, extending, and pushing), processing and handling movements (throwing, catching, and kicking). (24:75-76) (9:106-107) (19:43) (14:18) (17:45)

As exercises reflect individual's motor activity; it - necessarily - have beneficial effects on all aspects of an individual's life. it is the movements we choose for body composition and balance upbringing so acquiring flexibility that enables to perform large and wide extent movements in joints, and strengthens its different parts in balanced and consistent way, i.e. not to strengthen one at the expense of another, and increase benefit of good material in blood so that each member do his job in the best way possible. $(8: 38)$

From what mentioned above and through literature review and as a result of researchers visits to some mental education schools in Alexandria and interview those who taught physical education to see motor programs provided to mental disabled pupils) and inquire about the motor performance constraints and pilot studies which have shown lack of content of motor and physical exercises programs describing performance to develop motor skills for mentally disabled pupils), physical education teachers pointed significant low performance level in basic motor skills within this category, which called for conducting this study for the purpose of identifying the impact of physical exercise in developing some physical abilities and improve performance of some basic motor skills to mentally disabled students

\section{Research objectives:}

Identify Impact of physical exercise in developing some motor abilities and improving performance of some basic motor skills for mentally disabled pupils (8-12 years)

\section{Research hypotheses:}

- Proposed physical exercise program affect in developing some motor abilities for mentally disabled pupils (8-12 years)

- Proposed physical exercise program affect in improving performance of some basic motor skills for mentally disabled pupils (8-12 years)

\section{Research procedures:}

Research Methodology: Experimental approach was used for its suitability to research nature.

Human domain: This study was conducted on a sample of mentally disabled students in age stage ( 8 -12 years) with mental age 
ranges between (4-6) years and IQ range between $(50$ - 75) degrees.

Time domain: Pilot studies and premeasurements in the period from 26/01/2013 to $07 / 02 / 2013$, and main experiment in the period from $09 / 02 / 2013$ to $04 / 04 / 2013$, and post-measurements in the period from 06/04/2013 to $08 / 4 / 2013$.

Spatial domain: Saad Zaghloul and Ikhlas mental education schools, West Educational Department, Alexandria governorate .

Research Sample: Main study conducted on intentionally selected sample from mentally disables pupils from Saad Zaghloul mental education school, totaled to (20) students, divided into two groups, one experimental, and the other control each of (10) pupils, while the pilot studies conducted on intentionally selected sample from mentally disables pupils from Ikhlas mental education school totaled to (12) pupils.

Tests and measurements:

Mental measurements and tests:

Non-verbal Pictured IQ-test for measuring IQ. (25)

\section{Motor abilities tests for mentally disabled:}

Test battery prepared by HPERD for mentally disabled (8-18 years) for American National Association for mentally disabled which contain the following tests. (22)

It includes the following tests:

\section{1 - Flexed arm hang}

(flexion jang catching from top) for the longest period of stability.

\section{2 - Sit-Up}

(Lying) lift the trunk high to touch specific mark.

3 - Shuttle-Run
(Front standing position; Facing two wood cube 10 meters far) running forward to bring first cube and then return to bring the second one.

4 - Standing Broad Jump

(Standing) jump forward to the farthest distance

$$
5 \text { - } 50 \text { Yard Dash }
$$

(Standing) Running forward around playground, for a distance of 50 yards.

6 - Softball throwing for distance

(Standing, Caught hockey ball in hand) throw the ball from the top of the head to the farthest distance.

\section{7- 300 Yard run and walk}

(Standing) Running forward around playground, for a distance of 300 yards.

Physical tests with skill indicators for mentally disabled:

- Coordination tests:

- Coordination between eye and hand: Throw and receive a ball. (16:410)

- Coordination between legs and eyes: Numbered circles test. (26)

\section{1 - Throw and receive a ball.}

(Standing. Facing a wall at a distance of 1.5 meters away. Caught handball ball in hands) throw the ball on the wall and catch it after rebound from wall by hands.

\section{2 - Numbered circles test.}

(Standing. Facing a series of numbered circles drawn on the ground) jump into numbered circles.

\section{Balancing test:}

- Stable Balance: - long standing with Feet on the bar. (16: 434) 
- Motor balance: - walking test on the Swedish seat. (16: 429)

\section{1 - Long standing with Feet on the bar.}

(Forward Standing; high on a balance bar, Stability of the waist), Stable for longest possible time.

\section{2 - Walking test on the Swedish seat.}

(High Standing on Swedish seat) Walk forward to the end of seat, return to change direction and then return to the beginning of the seat.

\section{- Agility test: -}

Zigzag Running. (16: 357)

\section{1 - Zigzag running Test}

(Standing. Facing a set of colored cones on equal distances of $180 \mathrm{~cm}$ ) forward zigzag run on colored line drawn on the ground between the cones.

\section{Basic motor skills tests:}

1. Walking test (walking for $20 \mathrm{~m}$ distance). (7: 55)

2. Running test (running for $30 \mathrm{~m}$ distance). (7: 55)

3. Push test (medical ball pushing). (18: 414)

4. Throw and catch (throw with one hand inside squares on a wall and catch with both hands). (7: 55)

5. Throw test (throwing hockey ball $\mathrm{t}$ farthest distance). (7: 55)

\section{Test Standardizing:}

\section{Tests Validity:}

Comparison validity test between upper and lower quartiles carried out after applying test on pilot study sample (12 pupils)

Table (1)

differences between Highest and lowest quartiles in basic variables

\begin{tabular}{|c|c|c|c|c|c|c|c|c|}
\hline \multirow{2}{*}{ Variables } & \multirow{2}{*}{$\begin{array}{r}\text { Statistical } \\
\text { indicators }\end{array}$} & \multicolumn{2}{|c|}{ Highest quartile } & \multicolumn{2}{|c|}{ Lowest quartile } & \multirow{2}{*}{$\begin{array}{c}\text { Mean } \\
\text { Differences }\end{array}$} & \multirow{2}{*}{ T value } & \multirow{2}{*}{$\begin{array}{l}\text { Validity } \\
\text { coefficient }\end{array}$} \\
\hline & & Mean & \pm SD & Mean & $\pm \mathbf{S D}$ & & & \\
\hline \multirow{5}{*}{$\begin{array}{c}\text { Basic } \\
\text { Variables }\end{array}$} & Height (cm) & 131.204 & 5.843 & 130.153 & 4.935 & 1.051 & 0.014 & 0.004 \\
\hline & Weight (kg) & 30.146 & 3.261 & 29.754 & 3.671 & 0.392 & 0.196 & 0.062 \\
\hline & $\begin{array}{c}\text { Chronological age } \\
\text { (years) }\end{array}$ & 10.527 & 0.985 & 10.322 & 0.749 & 0.205 & 0.406 & 0.127 \\
\hline & Mental age (years) & 5.367 & 0.934 & 5.142 & 0.679 & 0.225 & 0.478 & 0.149 \\
\hline & IQ score & 71.936 & 7.929 & 56.149 & 6.58 & 15.787 & 3.753* & 0.765 \\
\hline
\end{tabular}

* Significant at the 0.05 level $=2.23$

Table (1) results that sample are equal in height, weight, chronological age and mental age variables, as $(\mathrm{T})$ value ranged between $(0.014,0.478)$, and these values are less than the significant $\mathrm{T}$ value at level of $(0.5)$, while that there were difference between highest and lowest quartiles in IQ variable as (T) value was $(3.753 *)$ which is higher than significant value at $(0.5)$ which confirms that the category, which was the basis of choosing highest and lowest quartiles is true. 
Table (2)

differences between Highest and lowest quartiles in HPERD battery for Mentally disabled

\begin{tabular}{|c|c|c|c|c|c|c|c|c|}
\hline \multirow{2}{*}{\multicolumn{2}{|c|}{ Variables }} & \multicolumn{2}{|c|}{ Highest quartile } & \multicolumn{2}{|c|}{ Lowest quartile } & \multirow{2}{*}{$\begin{array}{c}\text { Mean } \\
\text { Differences }\end{array}$} & \multirow{2}{*}{$\begin{array}{c}\text { T } \\
\text { value }\end{array}$} & \multirow{2}{*}{$\begin{array}{l}\text { Validity } \\
\text { coefficient }\end{array}$} \\
\hline & & Mean & $\pm \mathbf{S D}$ & Mean & \pm SD & & & \\
\hline \multirow{7}{*}{$\begin{array}{c}\text { AAHPERD } \\
\text { test for } \\
\text { mentally } \\
\text { disables }\end{array}$} & $\begin{array}{c}\text { Flexed arm hang } \\
\text { (second) }\end{array}$ & 8.205 & 2.265 & 2.265 & 1.431 & 5.94 & $6.030 *$ & 0.886 \\
\hline & $\begin{array}{c}\text { Sit-Up } \\
\text { (number/minute) }\end{array}$ & 13.745 & 8.215 & 8.215 & 1.374 & 5.53 & $7.191 *$ & 0.915 \\
\hline & Shuttle-Run (second) & 27.521 & 37.189 & 37.189 & 2.985 & 9.668 & $5.327 *$ & 0.86 \\
\hline & $\begin{array}{c}\text { Standing Broad Jump } \\
(\mathrm{cm})\end{array}$ & 75.201 & 57.231 & 57.231 & 7.148 & 17.97 & $5.055 *$ & 848 \\
\hline & $\begin{array}{c}50 \text { Yard Dash } \\
\text { (second) }\end{array}$ & 15.342 & 21.294 & 21.294 & 1.954 & 5.952 & $6.463 *$ & 0.898 \\
\hline & $\begin{array}{c}\text { Softball throwing for } \\
\text { distance }(\mathrm{m})\end{array}$ & 16.247 & 8.978 & 8.978 & 1.571 & 7.269 & $8.095 *$ & 0.931 \\
\hline & $\begin{array}{c}300 \text { Yard run and } \\
\text { walk (second) }\end{array}$ & 174.211 & 11.723 & 208.536 & 11.723 & 34.325 & $5.483 *$ & 0.866 \\
\hline
\end{tabular}

* Significant at the 0.05 level $=2.23$

Table (2) results reveal statistically significant value at (0.05) level, validity significance differences between the two coefficient ranged between $(0.848,0.931)$ groups as $(\mathrm{T})$ value ranged between $(6.030 *$, which confirms that the battery tests measure $\left.8.095^{*}\right)$, which are relatively high from $(\mathrm{T})$ what it designed for.

Table (3)

differences between Highest and lowest quartiles in physical tests (with skill Indicators)

\begin{tabular}{|c|c|c|c|c|c|c|c|c|}
\hline \multirow[b]{2}{*}{ Variables } & \multirow{2}{*}{$\begin{array}{l}\text { Statistical } \\
\text { indicators }\end{array}$} & \multicolumn{2}{|c|}{$\begin{array}{l}\text { Highest } \\
\text { quartile }\end{array}$} & \multicolumn{2}{|c|}{ Lowest quartile } & \multirow{2}{*}{ 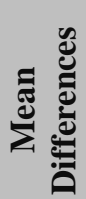 } & \multirow{2}{*}{$\underset{\mathfrak{E}}{\stackrel{0}{\Xi}}$} & \multirow{2}{*}{ 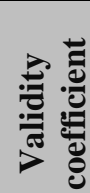 } \\
\hline & & Mean & $\pm \mathbf{S D}$ & Mean & \pm SD & & & \\
\hline $\begin{array}{c}\text { Coordination } \\
\text { between eye and } \\
\text { hand }\end{array}$ & $\begin{array}{l}\text { Amended Throw and } \\
\text { receive a ball. } \\
\text { (number/30 seconds) }\end{array}$ & 9.624 & 1.535 & 4.159 & 1.256 & 5.465 & $6.747 *$ & 0.905 \\
\hline $\begin{array}{c}\text { Coordination } \\
\text { between legs and } \\
\text { hand }\end{array}$ & $\begin{array}{l}\text { Numbered circles test. } \\
\text { (second) }\end{array}$ & 6.453 & 0.634 & 7.829 & 0.689 & 1.376 & $3.602 *$ & 0.751 \\
\hline Stable Balance & $\begin{array}{l}\text { long standing with Feet } \\
\text { on the bar (second) }\end{array}$ & 29.626 & 2.426 & 21.293 & 2.322 & 8.333 & 6.078* & 0.887 \\
\hline Motor balance & $\begin{array}{l}\text { walking on the Swedish } \\
\text { seat (seconds) }\end{array}$ & 5.585 & 0.269 & 6.893 & 0.684 & 1.308 & $4.36^{*}$ & 0.809 \\
\hline Agility & $\begin{array}{l}\text { Zigzag Running } \\
\text { (seconds) }\end{array}$ & 12.239 & 0.814 & 16.471 & 0.972 & 4.232 & $8.170 *$ & 0.933 \\
\hline
\end{tabular}

Table (3) results reveal statistically significant value at (0.05) level, validity significance differences between the two coefficient ranged between $(0.751,0.933)$ groups as $(\mathrm{T})$ value ranged between $(3.602 *$, which confirms that tests measure what it $\left.8.170^{*}\right)$, which are relatively high from $(\mathrm{T})$ designed for. 
Table (4)

differences between Highest and lowest quartiles in basic motor skills tests

\begin{tabular}{|c|c|c|c|c|c|c|c|c|}
\hline \multirow[b]{2}{*}{ Variables } & \multirow[t]{2}{*}{ Statistical indicators } & \multicolumn{2}{|c|}{$\begin{array}{l}\text { Highest } \\
\text { quartile }\end{array}$} & \multicolumn{2}{|c|}{ Lowest quartile } & \multirow{2}{*}{ 冚 } & \multirow{2}{*}{ 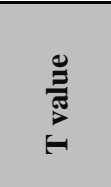 } & \multirow{2}{*}{ 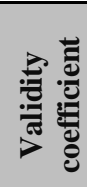 } \\
\hline & & Mean & $\pm \mathbf{S D}$ & Mean & $\pm \mathbf{S D}$ & & & \\
\hline \multirow{3}{*}{$\begin{array}{c}20 \mathrm{~m} \\
\text { walking } \\
\text { (second) }\end{array}$} & 20 m walking time & 12.149 & 0.985 & 18.204 & 1.193 & 6.055 & $9.581 *$ & 0.95 \\
\hline & No. of steps & 29.328 & 1.379 & 33.824 & 1.245 & 4.496 & $5.931 *$ & 0.882 \\
\hline & Step time average & 0.305 & 0.039 & 0.835 & 0.066 & 0.63 & $3.913 *$ & 0.778 \\
\hline \multirow{3}{*}{$\begin{array}{l}30 \mathrm{~m} \\
\text { running } \\
\text { (second) }\end{array}$} & $30 \mathrm{~m}$ running time & 12.192 & 0.985 & 18.437 & 1.289 & 6.245 & $9.434 *$ & 0.948 \\
\hline & No. of steps & 19.521 & 0.849 & 22.341 & 1.145 & 0.582 & $4.845 *$ & 0.837 \\
\hline & Step time average & 0.601 & 0.039 & 0.889 & 0.097 & 0.208 & $3.782 *$ & 0.767 \\
\hline Push & $\begin{array}{l}\text { Medical ball push by hands to } \\
\text { roll on ground to farthest } \\
\text { distance }(\mathrm{m})\end{array}$ & 29.527 & 2.921 & 9.423 & 1.327 & 20.104 & $\begin{array}{c}15.347 \\
*\end{array}$ & 0.979 \\
\hline \multirow{3}{*}{$\begin{array}{l}\text { Amended } \\
\text { Throwing and } \\
\text { catching test }\end{array}$} & Throwing (marks) & 13.937 & 0.602 & 8.178 & 0.974 & 5.759 & $\begin{array}{c}12.332 \\
*\end{array}$ & 0.969 \\
\hline & Catching (marks) & 12.204 & 0.644 & 7.521 & 0.856 & 4.683 & $\begin{array}{c}10.716 \\
*\end{array}$ & 0.959 \\
\hline & $\begin{array}{c}\text { Total of throwing and catching } \\
\text { marks }\end{array}$ & 26.141 & 0.849 & 15.699 & 0.948 & 10.442 & $\begin{array}{c}20.081 \\
*\end{array}$ & 0.988 \\
\hline \multirow{2}{*}{$\begin{array}{l}\text { Hockey ball } \\
\text { throwing for } \\
\text { distance }\end{array}$} & Right hand & 14.158 & 1.625 & 7.298 & 0.952 & 6.86 & $8.921 *$ & 0.943 \\
\hline & Left hand & 11.376 & 0.987 & 4.831 & 0.782 & 6.545 & $\begin{array}{c}12.808 \\
*\end{array}$ & 0.971 \\
\hline
\end{tabular}

* Significant at the 0.05 level $=\mathbf{2 . 2 3}$

Table (4) results reveal statistically significance differences between the two groups as $(\mathrm{T})$ value ranged between $\left(3.782^{*}\right.$, $20.081 *)$, which are relatively high from $(\mathrm{T})$ significant value at (0.05) level, validity coefficient ranged between $(0.767,0.988)$ which confirms that tests measure what it designed for.

\section{Tests Reliability:}

The reliability coefficient was calculated using test - retest method on pilot study sample (12 mentally disabled students) with seven days interval between the two tests. 
Table (5)

Differences between the first and second test in HPERD battery for mentally disabled

\begin{tabular}{|c|c|c|c|c|c|c|c|c|}
\hline \multirow{2}{*}{ Variables } & & \multicolumn{2}{|c|}{ First test } & \multicolumn{2}{|c|}{ Second test } & \multirow{2}{*}{$\begin{array}{c}\text { Mean } \\
\text { Differences }\end{array}$} & \multirow{2}{*}{$\begin{array}{c}\mathbf{T} \\
\text { value }\end{array}$} & \multirow{2}{*}{$\begin{array}{l}\text { Reliability } \\
\text { coefficient }\end{array}$} \\
\hline & & Mean & $\pm \mathbf{S D}$ & Mean & $\pm \mathbf{S D}$ & & & \\
\hline \multirow{7}{*}{$\begin{array}{c}\text { AAHPERD } \\
\text { test for } \\
\text { mentally } \\
\text { disables }\end{array}$} & $\begin{array}{l}\text { Flexed arm hang } \\
\text { (second) }\end{array}$ & 6.528 & 4.852 & 6.289 & 4.729 & 0.239 & 0.904 & 0.897 \\
\hline & $\begin{array}{c}\text { Sit-Up } \\
\text { (number/minute) }\end{array}$ & 13.467 & 2.645 & 13.127 & 2.821 & 0.34 & 0.952 & 0.815 \\
\hline & Shuttle-Run (second) & 32.895 & 6.839 & 33.215 & 6.723 & 0.32 & 1.143 & 0.942 \\
\hline & $\begin{array}{c}\text { Standing Broad Jump } \\
(\mathrm{cm})\end{array}$ & 66.343 & 13.254 & 64.624 & 13.371 & 1.719 & 1.295 & 0.859 \\
\hline & $\begin{array}{c}50 \text { Yard Dash } \\
\text { (second) }\end{array}$ & 17.482 & 3.295 & 17.835 & 3.536 & 0.353 & 1.372 & 0.769 \\
\hline & $\begin{array}{l}\text { Softball throwing for } \\
\text { distance (m) }\end{array}$ & 13.165 & 4.446 & 13.094 & 4.205 & 0.071 & 0.695 & 0.815 \\
\hline & $\begin{array}{l}300 \text { Yard run and } \\
\text { walk (second) }\end{array}$ & 190.849 & 20.156 & 192.943 & 21.954 & 2.094 & 1.349 & 0.806 \\
\hline
\end{tabular}

* Significant at the 0.05 level $=2.23$

Table (5) reveal no significant difference (T) significant value at (0.05) level, and between the two tests as $(\mathrm{T})$ value ranged reliability coefficient ranged between $(0.769$ between $(0.695-1,372)$, which is lower than 0.942) which confirms tests reliability.

Table (6)

Differences between the first and second test in physical tests (with skill Indicators)

\begin{tabular}{|c|c|c|c|c|c|c|c|c|}
\hline \multirow{2}{*}{\multicolumn{2}{|c|}{ Variables }} & \multicolumn{2}{|c|}{$\begin{array}{l}\text { Highest } \\
\text { quartile }\end{array}$} & \multicolumn{2}{|c|}{$\begin{array}{l}\text { Lowest } \\
\text { quartile }\end{array}$} & \multirow{2}{*}{ 龸 } & \multirow{2}{*}{$\underset{F}{\stackrel{0}{ٍ}}$} & \multirow{2}{*}{ 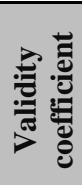 } \\
\hline & & Mean & $\pm \mathrm{SD}$ & Mean & $\pm \mathrm{SD}$ & & & \\
\hline $\begin{array}{c}\text { Coordination between } \\
\text { eye and hand }\end{array}$ & $\begin{array}{l}\text { Amended Throw and receive a } \\
\text { ball. (number/30 seconds) }\end{array}$ & 6.751 & 3.204 & 6.602 & 3.114 & 0.113 & 0.298 & 0.922 \\
\hline $\begin{array}{c}\text { Coordination between } \\
\text { legs and hand }\end{array}$ & Numbered circles test. (second) & 6.732 & 0.743 & 7.035 & 0.845 & 0.303 & 0.954 & 0.876 \\
\hline Stable Balance & $\begin{array}{l}\text { long standing with Feet on the } \\
\text { bar (second) }\end{array}$ & 27.092 & 4.543 & 26.964 & 4.605 & 0.128 & 0.716 & 0.965 \\
\hline Motor balance & $\begin{array}{c}\text { walking on the Swedish seat } \\
\text { (seconds) }\end{array}$ & 5.634 & 0.645 & 5.549 & 0.635 & 0.085 & 0.643 & 0.885 \\
\hline Agility & Zigzag Running (seconds) & 13.729 & 2.204 & 13.895 & 2.165 & 0.166 & 1.245 & 0.891 \\
\hline
\end{tabular}

* Significant at the $\mathbf{0 . 0 5}$ level $=\mathbf{2 . 2 3}$

Table (6) reveal no significant difference (T) significant value at (0.05) level, and between the two tests as $(\mathrm{T})$ value ranged reliability coefficient ranged between $(0.876$ between (0.298-1.245), which is lower than 0.922) which confirms tests reliability. 
Table (7)

Differences between the first and second test in basic motor skill tests

\begin{tabular}{|c|c|c|c|c|c|c|c|c|}
\hline \multirow[b]{2}{*}{ Variables } & \multirow[t]{2}{*}{ Statistical indicators } & \multicolumn{2}{|c|}{$\begin{array}{l}\text { Highest } \\
\text { quartile }\end{array}$} & \multicolumn{2}{|c|}{ Lowest quartile } & \multirow{2}{*}{ 苋 } & \multirow{2}{*}{ 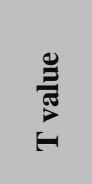 } & \multirow{2}{*}{ 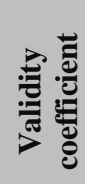 } \\
\hline & & Mean & $\pm \mathbf{S D}$ & Mean & \pm SD & & & \\
\hline \multirow{3}{*}{$\begin{array}{c}20 \mathrm{~m} \\
\text { walking } \\
\text { (second) }\end{array}$} & 20 m walking time & 14.514 & 2.743 & 14.502 & 3.029 & 0.012 & 0.364 & 0.889 \\
\hline & No. of steps & 31.502 & 2.276 & 31.515 & 2.652 & 0.013 & 0.109 & 0.735 \\
\hline & Step time average & 0.465 & 0.084 & 0.468 & 0.76 & 0.003 & 0.715 & 0.988 \\
\hline \multirow{3}{*}{$\begin{array}{l}30 \mathrm{~m} \\
\text { running } \\
\text { (second) }\end{array}$} & $30 \mathrm{~m}$ running time & 14.302 & 3.298 & 14.712 & 3.437 & 0.41 & 0.991 & 0.917 \\
\hline & No. of steps & 20.267 & 1.665 & 20.397 & 1.698 & 0.108 & 0.954 & 0.958 \\
\hline & Step time average & 0.715 & 0.133 & 0.722 & 0.141 & 0.007 & 0.553 & 0.934 \\
\hline Push & $\begin{array}{l}\text { Medical ball push by hands to roll } \\
\text { on ground to farthest distance }(\mathrm{m})\end{array}$ & 18.891 & 12.204 & 17.258 & 11.984 & 1.633 & 1.143 & 0.854 \\
\hline \multirow{3}{*}{$\begin{array}{c}\text { Amended } \\
\text { Throwing and } \\
\text { catching test }\end{array}$} & Throwing (marks) & 10.821 & 3.102 & 10.493 & 3.285 & 0.328 & 0.798 & 0.885 \\
\hline & Catching (marks) & 9.723 & 2.642 & 9.541 & 2.533 & 0.182 & 0.976 & 0.935 \\
\hline & $\begin{array}{c}\text { Total of throwing } \\
\text { and catching marks }\end{array}$ & 20.544 & 5.682 & 20.034 & 5.592 & 0.51 & 0.945 & 0.914 \\
\hline \multirow{2}{*}{$\begin{array}{l}\text { Hockey ball } \\
\text { throwing for } \\
\text { distance }\end{array}$} & Right hand & 10.927 & 3.531 & 10.442 & 3.141 & 0.485 & 0.996 & 0.885 \\
\hline & Left hand & 7.849 & 3.431 & 7.652 & 3.367 & 0.197 & 0.917 & 0.886 \\
\hline
\end{tabular}

* Significant at the 0.05 level $=2.23$

Table (7) reveal no significant difference Using SPSS the following statistical between the two tests as $(\mathrm{T})$ value ranged coefficients and tests carried out and between (0.109-1.143), which is lower than (T) significant value at (0.05) level, and reliability coefficient ranged between $(0.735$ 0.988) which confirms tests reliability. calculated:

Mean - standard deviation - skewness factor - percentage - $(\mathrm{T})$ value - correlation coefficient

\section{Statistical work:}

Table (8)

Statistical description of study sample in basic variables prior to experiment $(n=20)$

\begin{tabular}{|l|c|c|c|c|c|}
\hline \multicolumn{2}{|r|}{$\begin{array}{r}\text { Statistical } \\
\text { indicators }\end{array}$} & \multicolumn{5}{|c|}{ Description indicators } \\
\cline { 3 - 6 } Variables & & Mean & \pm SD & Skewness coefficient & $\begin{array}{c}\text { variation coefficient } \\
\%\end{array}$ \\
\hline & Height (cm) & 135.612 & 7.485 & 0.082 & 5.519 \\
\cline { 2 - 6 } Basic Variables & Weight (kg) & 33.449 & 6.288 & 0.135 & 18.799 \\
\cline { 2 - 6 } & Chronological age (years) & 10.537 & 1.205 & 0.641 & 11.436 \\
\cline { 2 - 6 } & Mental age (years) & 5.102 & $\mathbf{0 . 7 1 3}$ & $\mathbf{- 0 . 0 5 4}$ & 13.975 \\
\cline { 2 - 6 } & IQ score & 54.639 & 3.273 & 1.541 & 5.99 \\
\hline
\end{tabular}


Table (8) results reveal that skewness for all basic variables is between $( \pm 3)$, which indicates that all basic variables for study sample are under normal distribution curve, results also reveal that of coefficient of variation value for all basic variables is less than 20\%, which indicates sample homogeneity in all the variables under consideration.

\section{Homogeneous between the two groups:}

Researchers insured homogeneous between the two groups in each of the basic variables (Age - Height - Weight - IQ- mental age) as well as in physical and skill tests under discussion.

Table (9)

differences significance between experimental and control groups in basic variables before experiment

\begin{tabular}{|c|c|c|c|c|c|c|c|}
\hline \multirow{2}{*}{ Variables } & \multirow{2}{*}{$\begin{array}{r}\text { Statistical } \\
\text { indicators }\end{array}$} & \multicolumn{2}{|c|}{$\begin{array}{c}\text { Experimental group } \\
(\mathrm{n}=10)\end{array}$} & \multicolumn{2}{|c|}{$\begin{array}{c}\text { Control group } \\
(\mathrm{n}=10)\end{array}$} & \multirow{2}{*}{$\begin{array}{c}\text { Mean } \\
\text { Differences }\end{array}$} & \multirow{2}{*}{ T value } \\
\hline & & Mean & \pm SD & Mean & \pm SD & & \\
\hline \multirow{5}{*}{$\begin{array}{c}\text { Basic } \\
\text { Variables }\end{array}$} & Height (cm) & 135.924 & 8.431 & $\mathbf{1 3 5 . 5 3 5}$ & 6.952 & 0.389 & 0.113 \\
\hline & Weight (kg) & 34.435 & 6.289 & 33.349 & 6.341 & 1.086 & 0.385 \\
\hline & Chronological age (years) & 10.792 & 1.068 & 10.435 & 1.235 & 0.357 & 0.692 \\
\hline & Mental age (years) & 5.792 & 0.613 & 5.269 & 0.678 & 0.142 & 0.491 \\
\hline & IQ score & 54.521 & 3.467 & 55.024 & 2.945 & 0.503 & 0.350 \\
\hline
\end{tabular}

* Significant at the 0.05 level $=\mathbf{2 . 1 0}$

Table (9) results reveal no statistically $(\mathrm{T})$ value ranged between $(0.113,0.692)$, significant differences at (0.05) level which are lower than (T) significant value at between experimental and control groups, as (0.05) level

Table (10)

differences significance between experimental and control groups in HPERD battery tests for mentally disabled before experiment

\begin{tabular}{|c|c|c|c|c|c|c|c|}
\hline \multirow{2}{*}{ Variables } & \multirow{2}{*}{$\begin{array}{r}\text { Statistical } \\
\text { indicators }\end{array}$} & \multicolumn{2}{|c|}{$\begin{array}{c}\text { Experimental group } \\
(n=10)\end{array}$} & \multicolumn{2}{|c|}{$\begin{array}{c}\text { Control group } \\
(\mathbf{n}=\mathbf{1 0})\end{array}$} & \multirow{2}{*}{$\begin{array}{c}\text { Mean } \\
\text { Differences }\end{array}$} & \multirow{2}{*}{ T value } \\
\hline & & Mean & $\pm \mathrm{SD}$ & Mean & $\pm \mathrm{SD}$ & & \\
\hline \multirow{7}{*}{$\begin{array}{l}\text { AAHPERD } \\
\text { test for } \\
\text { mentally } \\
\text { disables }\end{array}$} & Flexed arm hang (second) & 2.856 & 1.458 & 2.543 & 1.689 & 0.313 & 0.443 \\
\hline & Sit-Up (number/minute) & 11.426 & 1.307 & 11.924 & 1.452 & 0.498 & 0.806 \\
\hline & Shuttle-Run (second) & 37.932 & 3.315 & 37.561 & 3.921 & 0.371 & 0.228 \\
\hline & Standing Broad Jump (cm) & 58.796 & 4.965 & 56.949 & 4.453 & 1.847 & 1.312 \\
\hline & 50 Yard Dash (second) & 19.621 & 2.387 & 19.235 & 2.672 & 0.386 & 0.341 \\
\hline & $\begin{array}{c}\text { Softball throwing for } \\
\text { distance }(\mathrm{m})\end{array}$ & 9.451 & 1.446 & 9.768 & 1.798 & 0.317 & 0.434 \\
\hline & $\begin{array}{l}300 \text { Yard run and walk } \\
\text { (second) }\end{array}$ & 212.633 & 11.685 & 213.442 & 12.143 & 0.809 & 0.152 \\
\hline
\end{tabular}

Table (10) results reveal no statistically (T) value ranged between $(0.313,1.847)$, significant differences at (0.05) level which are lower than $(\mathrm{T})$ significant value at between experimental and control groups, as (0.05) level 
Table (11)

differences significance between experimental and control groups in physical tests (with skills indicators) before experiment

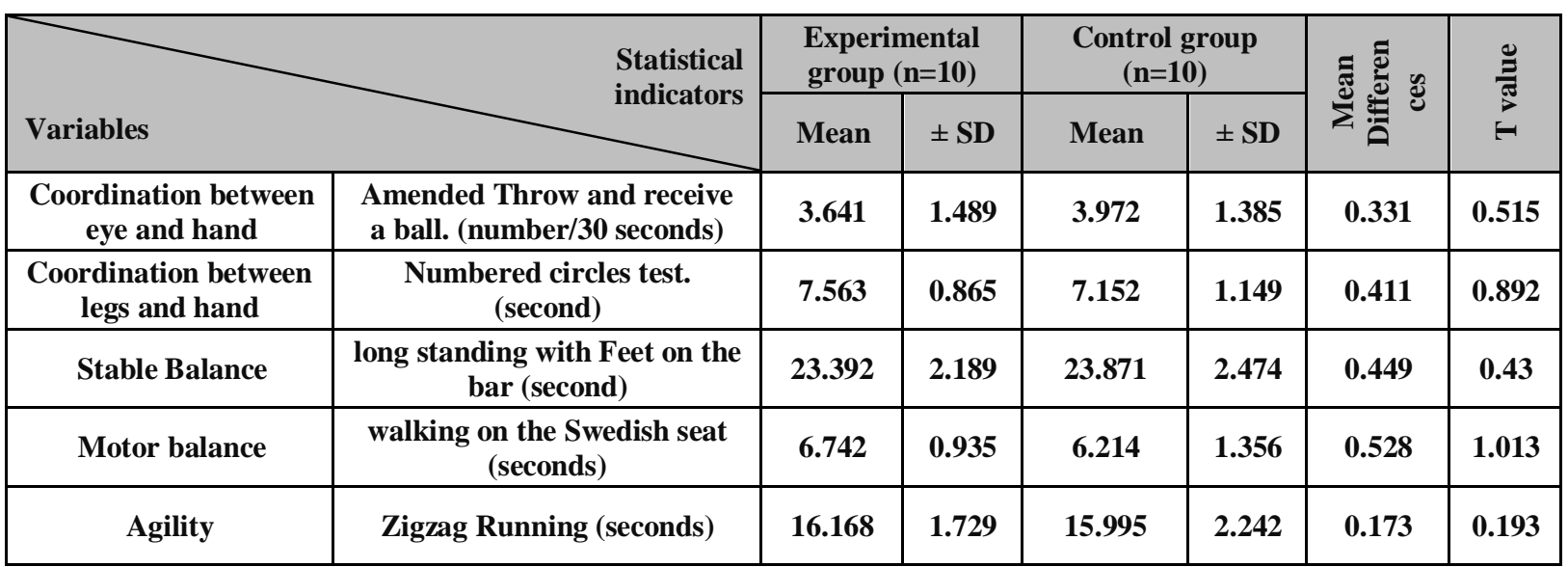

Table (11) results reveal no statistically (T) value ranged between $(0.193,1.013)$, significant differences at (0.05) level which are lower than $(\mathrm{T})$ significant value at between experimental and control groups, as (0.05) level

Table (12)

differences significance between experimental and control groups in basic motor skills tests before experiment

\begin{tabular}{|c|c|c|c|c|c|c|c|}
\hline \multirow{2}{*}{ Variables } & \multirow{2}{*}{$\underbrace{\text { Statistical indicators }}$} & \multicolumn{2}{|c|}{ Highest quartile } & \multicolumn{2}{|c|}{ Lowest quartile } & \multirow{2}{*}{$\begin{array}{c}\text { Mean } \\
\text { Differences }\end{array}$} & \multirow{2}{*}{$\begin{array}{c}\text { T } \\
\text { value }\end{array}$} \\
\hline & & Mean & \pm SD & Mean & \pm SD & & \\
\hline \multirow{3}{*}{$\begin{array}{c}20 \mathrm{~m} \\
\text { walking } \\
\text { (second) }\end{array}$} & $20 \mathrm{~m}$ walking time & 17.952 & 1.485 & 17.846 & 1.815 & 0.106 & 0.143 \\
\hline & No. of steps & 34.217 & 2.261 & 33.931 & 2.627 & 0.286 & 0.261 \\
\hline & Step time average & 0.539 & 0.033 & 0.528 & 0.054 & 0.011 & 0.55 \\
\hline \multirow{3}{*}{$\begin{array}{l}30 \mathrm{~m} \\
\text { running } \\
\text { (second) }\end{array}$} & $30 \mathrm{~m}$ running time & 15.814 & 2.843 & 16.271 & 3.134 & 0.457 & 0.342 \\
\hline & No. of steps & 21.252 & 3.317 & 21.965 & 3.918 & 0.713 & 0.439 \\
\hline & Step time average & 0.764 & 0.142 & 0.742 & 0.135 & 0.022 & 0.355 \\
\hline Push & $\begin{array}{l}\text { Medical ball push by hands to } \\
\text { roll on ground to farthest } \\
\text { distance }(\mathrm{m})\end{array}$ & 7.563 & 1.623 & 7.149 & 1.452 & 0.414 & 0.601 \\
\hline \multirow{3}{*}{$\begin{array}{l}\text { Amended } \\
\text { Throwing } \\
\text { and } \\
\text { catching test }\end{array}$} & Throwing (marks) & 7.891 & 1.258 & 8.287 & 1.565 & 0.396 & 0.624 \\
\hline & Catching (marks) & 7.825 & 1.319 & 8.514 & 1.1761 & 0.689 & 0.99 \\
\hline & $\begin{array}{c}\text { Total of throwing and catching } \\
\text { marks }\end{array}$ & 15.716 & 1.314 & 16.801 & 1.933 & 1.085 & 1.468 \\
\hline \multirow{2}{*}{$\begin{array}{l}\text { Hockey ball } \\
\text { throwing } \\
\text { for } \\
\text { distance }\end{array}$} & Right hand & 8.618 & 1.118 & 9.215 & 1.427 & 0.597 & 1.042 \\
\hline & Left hand & 4.235 & 0.991 & 4.503 & 1.214 & 0.268 & 0.54 \\
\hline
\end{tabular}

Table (12) results reveal no statistically which are lower than (T) significant value at significant differences at (0.05) level (0.05) level

between experimental and control groups, as (T) value ranged between $(0.550,1.468)$,
Tools and equipment used in measurements and tests: 
- (Restameter) to measure highest to nearest centimeter - Standardized medical scale to measure weight to nearest kilogram Handball balls - Hockey balls- Tennis balls balance bar to measure stable balance Swedish seats - measure tape, chalk, carton paper- Divided box- Stopwatch 1 / 100 compressed sponge mattress - wooden blocks - cones - scaled ruler - hoops or circles drawn on ground - spongy mattress sandbags - Swedish seats - Medical balls small colored balls - ropes - colored lines sticks.

\section{Pilot studies :}

Pilot studies conducted in order to:

- Identify the most important basic motor skills for mentally disabled students (8-12 years), and resulted in design for basic skills questionnaire presented to group of experts and specialists in the field of mental disabilities and physical education.

- Identify the most important physical abilities of basic motor skills for mentally disabled (8 - 12 years).

- Inventory of tools and facilities available in the school.

- Ensure the validity of tools used in measurement and implementation of the program.

- Identify tools and measuring equipment used.

- Ensure appropriate tests for the research community.

- Standardization legalization of tests that have been selected and to find scientific transactions for these tests.

- Determine the content of the proposed program exercises.
- Specify a time period for the program and the number of training units and number of units during the week and the time of each unit.

- Standardizing proposed program exercises and training units by determining average time performance of each exercise and average intra-rest time between each exercise and the other, and the average performance time form for each exercise model.

\section{Main study:}

Proposed training program for developing physical abilities for basic motor skills within mentally disabled ( 8 -12 years) distributed over (8) consecutive weeks with three (3) training units per week, training unit time was (40) minutes, Study began by program application as follows:

\section{Experimental group:}

- Trained with proposed training program consists of (24) units applied in training days (Sunday - Tuesday Thursday) for (2) months.

\section{Control group:}

- Trained with school motor curriculum through physical education classes on (Sunday - Tuesday - Thursday) days for (2) months too.

The proposed program:

\section{Program Content:}

\section{Training unit components:}

- Warm-up: duration (7) minutes, contains exercises in the form of games aiming to general preparation of muscles and stimulates blood circulation and preparing body joints to accept effort. 
- The main part: duration (30 minutes), includes physical exercises to improve some of basic motor skills.

- Final: duration (3) minutes, contains cool-down exercises to return to nearly normal situation.

\section{Program development foundation:}

- Using low-intensity interval training method for physical abilities development.

- Using load-rest principle as a base for the exercise to develop necessary abilities for the students.

- Load gradual raise.

- Right repetition time of the exercise.

- Continue to perform exercise and training. (5:212) (13:170) (3:288) (2:178-179)

- Taken into account when developing and implementing the program that exercises to be in gradual training units.

- Training unit contains (3) warm-up exercises or barriers exercises (for functional preparation) carried out individually.
- Training units contains (6) physical preparation exercises (for developing physical abilities), exercise performance time (30 s), intra- rest time (40 s).

- Training units contains (2) cool-down exercises.

- Medium intensity has been used in physical preparation exercises, where it was from $50 \%$ to $70 \%$ of pupil's maximum level while performing exercises.

- Exercise was without tools and use body weight, with using the tools (Leather balls- Seats - hoopSandbags - wands).

\section{Standardizing exercises time:}

- Maximum performance time for each exercise was measured for each pupil individually; pupil's average time for performing each exercise was about 30 seconds.

- $50 \%$ of pupil's maximum repetition was calculated in performing exercises (see table 13) 
Table (13)

Physical preparation exercises time distribution

\begin{tabular}{|l|l|}
\hline \multicolumn{1}{|c|}{ Training foundations } & \multicolumn{1}{c|}{ Physical preparation } \\
\hline Number of physical preparation exercises main part & (6) exercises \\
\hline Exercise model time & (60) seconds \\
\hline Number of sets per exercise & (4) sets \\
\hline Time doing the exercise in the same group & (30) seconds \\
\hline Intra-rest for exercise in same group exercise & (40) SECONDS \\
\hline Overall performance time for exercise groups & (120) SECONDS \\
\hline Overall Intra-rest time for exercise groups & (120) SECONDS \\
\hline Intra-rest time between each exercise groups (model performance) & (60) SECONDS \\
\hline Total exercise time (performance + rest) & (5) MINUTES \\
\hline Number of training units / week & (3) TIMES \\
\hline Physical preparation exercises time in training unit & (30) MINUTES \\
\hline
\end{tabular}

\section{Results and discussion}

Table (14)

Differences significance between the experimental and control groups in HPERD battery tests for mentally disabled after experiment

\begin{tabular}{|c|c|c|c|c|c|c|c|}
\hline \multirow{2}{*}{ Variables } & \multirow[t]{2}{*}{$\begin{array}{l}\text { Statistical } \\
\text { indicators }\end{array}$} & \multicolumn{2}{|c|}{$\begin{array}{c}\text { Experimental } \\
\text { group } \\
(\mathbf{n}=\mathbf{1 0})\end{array}$} & \multicolumn{2}{|c|}{$\begin{array}{l}\text { Control group } \\
\qquad(\mathbf{n}=\mathbf{1 0})\end{array}$} & \multirow{2}{*}{$\begin{array}{c}\text { Mean } \\
\text { Differences }\end{array}$} & \multirow[t]{2}{*}{ T value } \\
\hline & & Mean & $\pm \mathbf{S D}$ & Mean & \pm SD & & \\
\hline \multirow{7}{*}{$\begin{array}{c}\text { AAHPERD } \\
\text { test for } \\
\text { mentally } \\
\text { disables }\end{array}$} & Flexed arm hang (second) & 9.452 & 2.432 & 3.873 & 1.803 & 5.579 & $5.830 *$ \\
\hline & Sit-Up (number/minute) & 16.914 & 2.115 & 12.921 & 1.354 & 3.993 & $8.926 *$ \\
\hline & Shuttle-Run (second) & 28.339 & 3.264 & 34.958 & 3.527 & 6.619 & $4.355 *$ \\
\hline & Standing Broad Jump (cm) & 76.927 & 4.913 & 60.147 & 4.656 & 16.78 & $7.841 *$ \\
\hline & 50 Yard Dash (second) & 14.562 & 1.252 & 18.163 & 1.314 & 3.601 & $6.274 *$ \\
\hline & $\begin{array}{l}\text { Softball throwing for distance } \\
(\mathrm{m})\end{array}$ & 16.341 & 2.119 & 11.925 & 1.721 & 4.416 & $5.177 *$ \\
\hline & $\begin{array}{c}300 \text { Yard run and walk } \\
\text { (second) }\end{array}$ & 179.251 & 11.712 & 202.103 & 12.215 & 22.852 & $4.270 *$ \\
\hline
\end{tabular}

* Significant at the 0.05 level $=2.10$

Table (14) results reveal existence of effectiveness of proposed physical exercise statistically significant differences at (0.05) program, which has had effective impact in level between the experimental and control groups after the experiment (program implementation) in favor of experimental group in all battery tests, (T) value ranged between $(4.270,8.926)$, which are larger than the $(\mathrm{T})$ significance value at (0.05) level. Researchers return this improvement to the developing some physical abilities, so first hypothesis could be accepted (Proposed physical exercise program affect in developing some motor abilities for mentally disabled pupils (8-12 years).

This is consistent with findings of Hisham Omar (1998) that is mentally disabled 
practicing of physical activities and motor skills lead to improved motor performance and realization of motor skills to prepare them for everyday life skills, motor skills and self-dependence in facing everyday life problems. (23:118)

It is also consistent with conclusion of Abdullah Saeed (2009) that the proposed introductory games for learnable mentally disabled in vocational rehabilitation phase has led to development of motor skills (explosive power - speed - Coordination motor reaction speed - performance speed). (26:108)

Results also consistent with findings of the Salah Essawi (1992) that practice of sports activity, which includes games programs contribute in raising physical fitness including speed for mentally disabled.(22:152)

Table (15)

Differences significance between the experimental and control groups in physical tests (with skill indicators

\begin{tabular}{|c|c|c|c|c|c|c|c|}
\hline \multirow{2}{*}{\multicolumn{2}{|c|}{$\begin{array}{ll}\text { Variables } & \begin{array}{c}\text { Statistical } \\
\text { indicators }\end{array} \\
\end{array}$}} & \multicolumn{2}{|c|}{$\begin{array}{c}\text { Experimental group } \\
(n=10)\end{array}$} & \multicolumn{2}{|c|}{$\begin{array}{c}\text { Control group } \\
(\mathbf{n}=\mathbf{1 0})\end{array}$} & \multirow{2}{*}{$\begin{array}{c}\text { Mean } \\
\text { Differences }\end{array}$} & \multirow{2}{*}{ T value } \\
\hline & & Mean & $\pm \mathrm{SD}$ & Mean & $\pm \mathbf{S D}$ & & \\
\hline $\begin{array}{l}\text { Coordination } \\
\text { between eye } \\
\text { and hand } \\
\end{array}$ & $\begin{array}{c}\text { Amended Throw and } \\
\text { receive a ball. (number/30 } \\
\text { seconds) } \\
\end{array}$ & 9.407 & 1.612 & 5.714 & 1.219 & 3.693 & 5.779* \\
\hline $\begin{array}{c}\text { Coordination } \\
\text { between legs } \\
\text { and hand }\end{array}$ & $\begin{array}{l}\text { Numbered circles test. } \\
\text { (second) }\end{array}$ & 6.013 & 0.857 & 6.925 & 0.727 & 0.912 & $2.569 *$ \\
\hline $\begin{array}{c}\text { Stable } \\
\text { Balance } \\
\end{array}$ & $\begin{array}{l}\text { long standing with Feet on } \\
\text { the bar (second) }\end{array}$ & 31.124 & 2.432 & 25.237 & 2.153 & 5.887 & $5.732 *$ \\
\hline $\begin{array}{c}\text { Motor } \\
\text { balance }\end{array}$ & $\begin{array}{c}\text { walking on the Swedish } \\
\text { seat (seconds) }\end{array}$ & 5.011 & 0.714 & 6.128 & 0.935 & 1.117 & 3.003* \\
\hline Agility & Zigzag Running (seconds) & 11.614 & 0.834 & 15.673 & 0.956 & 4.059 & $10.122 *$ \\
\hline
\end{tabular}

* Significant at the 0.05 level $=2.10$

Table (15) results reveal existence of statistically significant differences at (0.05) level between the experimental and control groups after the experiment (program implementation) in favor of experimental group in physical tests (with skill indicators), (T) value ranged between $(2.569,10.122)$, which are larger than the $(\mathrm{T})$ significance value at (0.05) level. Researchers return this improvement to the effectiveness of physical exercise in the proposed program, which was followed by experimental group, including varied and exciting exercises either free or with tools with gradual load led to development of some motor skills (coordination- balance - Agility), which reflected, hence its effect reflected on performance of some basic motor skills. So second hypothesis could be accepted (Proposed physical exercise program affect in improving performance of some basic motor skills for mentally disabled pupils (812 years).

This is consistent with what pointed out by Hisham Omar (1998) that (powerCoordination- Speed - Balance - Agilityreaction time) can be better developed through organized training programs, and also with what he concluded that mentally disabled children cannot arrive to their maximum in muscle coordination, motor skills, balance and loco motor perceptual except through organized physical education programs at home and at school.(23:120) 
Ahmed El Shazly (1995), Abdel Moneim performance, which is compound and Suleiman et al (1995) and Sobhy Hassanein difficult. Balance is an essential component (1996) emphasized that balance plays an of motor skills performance as walking, important role in success of skills running and leap.(11:120) (8:105) (15:238)

Table (16)

Differences significance between the experimental and control groups in basic motor skills tests (with skill indicators

\begin{tabular}{|c|c|c|c|c|c|c|c|}
\hline \multirow{2}{*}{\multicolumn{2}{|c|}{ Variables }} & \multicolumn{2}{|c|}{ Highest quartile } & \multicolumn{2}{|c|}{ Lowest quartile } & \multirow{2}{*}{$\begin{array}{c}\text { Mean } \\
\text { Differences }\end{array}$} & \multirow{2}{*}{ T value } \\
\hline & & Mean & \pm SD & Mean & \pm SD & & \\
\hline \multirow{3}{*}{$\begin{array}{l}20 \mathrm{~m} \\
\text { walking } \\
\text { (second) }\end{array}$} & $20 \mathrm{~m}$ walking time & 12.415 & 1.137 & 17.013 & 1.913 & 4.598 & $6.531 *$ \\
\hline & No. of steps & 30.012 & 1.923 & 33.725 & 2.258 & 3.713 & 3.958* \\
\hline & Step time average & 0.415 & 0.051 & 0.516 & 0.047 & 0.101 & $4.591 *$ \\
\hline \multirow{3}{*}{$\begin{array}{l}30 \mathrm{~m} \\
\text { running } \\
\text { (second) }\end{array}$} & $30 \mathrm{~m}$ running time & 10.914 & 1.205 & 15.612 & 1.918 & 4.698 & $6.799 *$ \\
\hline & No. of steps & 19.103 & 1.786 & 20.927 & 2.367 & 1.824 & 1.945 \\
\hline & Step time average & 0.551 & 0.071 & 0.714 & 0.108 & 0.163 & $3.976 *$ \\
\hline Push & $\begin{array}{l}\text { Medical ball push by hands to } \\
\text { roll on ground to farthest } \\
\text { distance (m) } \\
\end{array}$ & 24.389 & 2.914 & 16.253 & 2.132 & 8.136 & $7.124 *$ \\
\hline \multirow{3}{*}{$\begin{array}{l}\text { Amended } \\
\text { Throwing } \\
\text { and } \\
\text { catching test }\end{array}$} & Throwing (marks) & 13.463 & 1.252 & 9.214 & 0.954 & 4.249 & $8.532 *$ \\
\hline & Catching (marks) & 12.752 & 1.417 & 9.728 & 1.721 & 3.024 & $4.289 *$ \\
\hline & $\begin{array}{c}\text { Total of throwing and catching } \\
\text { marks }\end{array}$ & 26.215 & 1.785 & 18.942 & 2.126 & 7.273 & $8.284 *$ \\
\hline \multirow{2}{*}{$\begin{array}{l}\text { Hockey ball } \\
\text { throwing } \\
\text { for } \\
\text { distance }\end{array}$} & Right hand & 15.423 & 2.123 & 10.392 & 1.814 & 5.031 & $5.698 *$ \\
\hline & Left hand & 10.804 & 0.919 & 5.913 & 0.857 & 4.819 & $12.320 *$ \\
\hline
\end{tabular}

* Significant at the 0.05 level $=2.10$

Table (16) results reveal existence of statistically significant differences at (0.05) level between the experimental and control groups after the experiment (program implementation) in favor of experimental group in physical tests (with skill indicators), (T) value ranged between $(1.945,12.320)$, which are larger than the (T) significance value at (0.05) level. While there was no significant difference in (No. of steps of 30 meter running) as ( $\mathrm{T})$ value was (1.945) which is lower than $(\mathrm{T})$ significance value at (0.05) level.

Researchers return this improvement to effectiveness of the proposed physical exercise program, which was applied to the experimental group, including walk, leap and throw exercises ... Etc. varied and exciting for this category, which in turn improved the physical abilities under discussion which has had greatest impact in improving motor skills of the research sample, while there was no significant differences between the two groups in (No. of steps of 30 meter running); researchers attribute this to delayed motor development for mentally disables and what they suffer from dispersion and disorders specially in running, they are less able to walk and run in the right way and they are delayed with as 
least two years from their normal peers, and that confirms acceptance of second hypothesis (Proposed physical exercise program affect in improving performance of some basic motor skills for mentally disabled pupils (8-12 years).

These results are in consistent with findings of the Mohamed Mersal (1993), that basic movements development relatively slow as long as there is no suitable exercise, while this development is rapid and more diverse if we train pupils in a timely manner.(7:130)

Gomaa Sobhy (2006) confirmed that children training with mental disability on motor skills and craftsmanship bring them progress in motor performance and helps them to learn cognitive mental skills, because a mentally disabled child delayed in sitting, standing, walking, jumping and running, so he needs to train to develop of motor balance and motor skills development in general. (28:16)

In this regard Abdul Hakim Elmatar (1996), and Hassan Alnoasrh (2010) agreed that practicing sports activities lead to handicapped integration with their peers without disabilities, and acceptance of others. As lack in motor performance lead to withdrawal of disabled from motor experience motor, and thus lead to fall down of their performance level in sports skills, which leads to non-selection by their peers to participate in the play, which in turn leads to social isolation and inactivity life.(10:18) (6:158)

\section{Conclusions:}

1. Experimental group which followed the proposed training program of physical exercise surpassed control group.
2. Developing motor skills for mentally disabled pupils have a positive impact in improving basic motor skills (Walking - running - throwing catching-pushing- balance)

3. Mentally disabled pupils practice building and special purpose exercises with or withut using tools and by following scientific foundations when implemented resulted in improved motor skills for pupils with mental disabilities.

4. School motor curriculum contributes in improving basic motor skills of the control group, where results showed a slight improvement in performance level of those skills.

\section{Recommendations:}

1. Physical exercise program to be applied to mentally disabled pupils through specialized Physical Education graduates.

2. It is necessary when planning mental schools programs to develop physical abilities programs and implement it for mentally disabled students because of its positive impact in improving performance of basic motor skills.

3. Providing tools used in physical education classes in mental schools.

4. There is need of further scientific studies for this age group in order to improve other basic motor skills. 


\section{References}

1. Abdel Ghany,Y.M. (2001): Impact of motor treatment program on some of motor abilities and stature deviations of learnable mentally retarded, unpublished Ph.D. thesis, Faculty of Physical Education, Tanta University.

2. Abdel-Khalek,E.M. (2005): athletic training, theories and applications, $12^{\text {th }}$ ed., Knowledge House, Alexandria.

3. Ahmed,B.M.(1999): Principles and theories of sports training, Arab Thought House, Cairo.

4. Alhjursy,A.M. (2002): Educational of mentally handicapped children, ${ }^{1 s t}$ ed., Arab Thought House, Cairo.

5. Allawi,M.H. (1994): Sports training science, $\mathbf{1 3}^{\text {th }}$ ed., Knowledge House, Alexandria.

6. Alnoasrh,H.M. (2010): Handicapped (introduction to physical rehabilitation), University Printing House, Alexandria.

7. Arbab,M.M. (1993): Impact of a proposed exercises program to develop some of the basic movements of mentally disabled pupils (6-9 years), PhD Thesis, Faculty of Physical Education for Men, Alexandria University.

8. Borhom,A.S. , Abu Namra,M.K. (1995): Encyclopedia of physical exercise, $2^{\text {nd }} \mathrm{ed}$., Dar ELFekr Publishing and Distribution.

9. Deborah, A.W . \& Chrarles,A.B (1995) :foundation of physical Education and sports New York Graw Hill.

10. ELMatar.A.B. (1996): Physical Education (for handicapped), Cultural Series for
Association of Physical Education and Sports (5).

11. El Shazly,A.F. (1995): Balancing rules in the sports field, Knowledge House, Alexandria.

12. ElSokary,K.I., Mahran,W., Fawzy,F. (2005): basic skills in physical education for kindergarten for normal and handicapped children, Dar El Wafaa, Alexandria.

13. Hammad,M.I. (1999): Modern sports training, planning, implementation and leadership, the Arab Thought House, Cairo.

14. Hammad,M.I. (1999): Motor Education and its applications for kindergarten and primary schools, Mokhtar establishment for publication and distribution, Cairo.

15. Hassanein,M.S.(1996): Physical competency model, $1^{\text {st }}$ ed., Arab Thought House, Cairo.

16. Hassanein,M.S. (2001): Evaluation and measurement in physical education, vol. 1, ${ }^{4 t h}$ ed. Arab Thought House, Cairo.

17. Khalil,M.E.(2000): Motor Education, Theory and Practice, Faculty of Physical Education for Boys, Mansoura University.

18. Kholy,A.A., Kamel,O.R. (1998): Motor Education for children, 5th ed. Arab Thought House, Cairo.

19. Kirchner, G.,\& Fishburne,G. (1995) :Physical Education for ELementary school children lowa W.C. B.Brown Publisher.

20. McIntyre,C. (2000): Importance of play for handicapped children, translated by 
Khaled Al Ameri, Farouk pusblishing House, $1^{\text {st }}$ Arabic edition, Cairo.

21. Ministry of Education, Higher Education sector, central management of basic education, General management of Special Education(2003): technical guidance and administrative instructions for mental education classes.

22. Naga,S.M. (1998): Impact of application of proposed program of basic movements of athletics for mentally disabled children on some motor cognitive abilities, Unpublished Master Thesis, Faculty of Physical Education for Men, Alexandria University.

23. Omar,H.E. (1998): development of basic movements associated with rhythmic gymnastics using accompanying rhythmic and its impact on motor remembering for the mentally retarded, unpublished Ph.D. thesis, Faculty of Physical Education for Men, Alexandria University.

24. Osman,M.A. (1994): Motor education and sports training, Dar El Qalam, Kuwait.

25. Saleh,A.Z. (1979): Pictured IQ test, Anglo Egyptian Bookshop, Cairo.

26. Sharif,A.S. (2009): Impact of introductory games program on some motor abilities and basic skills in football within mental schools children, Unpublished Master Thesis, Faculty of Physical Education for Men, Alexandria University.

27. Soliman,M.F. (2004): Impact of proposed motor recreational program on development of basic motor skills for mentally disabled children (6-12 years), unpublished Master Thesis, Faculty of
Physical Education, Helwan University, 2004.

28. Taha,G.S. (2006): proposed program for small games and its impact on improving performance of some basic motor skills and social behavior of mentally disabled students, master thesis, Faculty of Physical Education, Alexandria University. 
